\title{
La enseñanza universitaria en odontología durante la pandemia
}

\author{
Hilda Fabiola Castillo Sánchez
}

\begin{abstract}
Resumen
La pandemia por la covid-19 nos llevó a cambiar nuestras costumbres y hábitos, y no sólo sociales, también los escolares al migrar a una educación a distancia, a un aprendizaje y a una enseñanza diferentes a las que por muchos años se habían trabajado. Como profesora en la Licenciatura de Cirujano Dentista, me enfrenté a diversos escenarios que no conocía y que, en determinado momento, no sabía cómo manejar, por ejemplo, las plataformas y, además, los sentimientos encontrados y la incertidumbre, entre otros. Sin embargo, esta transición ha dejado experiencias muy significativas debido a la respuesta que han tenido los estudiantes y aunque también se han presentado otras tristes, reconozco que, gracias al apoyo de la universidad, de amigos profesores, de los estudiantes y de mi familia, pude encontrar una respuesta a este escenario.
\end{abstract}

Palabras clave: asignaturas, estudiantes, plataformas, educación, covid-19.

\section{UNIVERSITY TEACHING IN DENTISTRY DURING THE PANDEMIC}

\begin{abstract}
The covid-19 pandemic caused a change in our customs and habits, and not in the social context, but also at school. By migrating to distance education, learning and teaching processes changed from those that had been carried out for many years. As a professor in the Bachelor of Dental Surgeon, I was faced with many unknown scenarios that, at a certain moment, I did not know how to handle, for example, platforms and, also, mixed feelings and uncertainty, among others. However, this transition has left very significant experiences due to the students' response, and although sad experiences also occurred, I recognize that, thanks to the support of the university, teacher friends, students and my family, I was able to find an answer in this scenario.
\end{abstract}

Keywords: subjects, students, platforms, education, covid-19.

Recepción: 28/10/2020. Aprobación: 1/12/2020.

Dol: http://doi.org/10.22201/cuaieed.16076079e.2021.22.1.17 
"La enseñanza universitaria en odontología durante la pandemia"

Hilda Fabiola Castillo Sánchez

Vol. 22, Núm. 1, enero-febrero 2021

Revista Digital Universitaria

\section{Hilda Fabiola Castillo Sánchez}

fa537@hotmail.com

Cirujana Dentista por la Universidad Nacional Autónoma de México (UnAm) y la Facultad de Estudios Superiores Iztacala (FES IZTACALA). Maestra en Educación por la Universidad Tecnológica de México (UNITEC) y Doctora en Educación con Mención Honorífica por la Universidad Intercontinental (UIC). Profesora de Asignatura en la Facultad de Estudios Superiores Iztacala en la Licenciatura de Cirujano Dentista desde hace 11 años.

Ha participado en diversos comités académicos, como instructora en diplomados en la Facultad de Estudios Superiores Iztacala y ponente en un evento internacional y en diversos nacionales en el área educativa.

E propósito de este documento es compartirles mi experiencia como profesora universitaria en la Licenciatura de Cirujano Dentista, en la Universidad Nacional Autónoma de México (UNAM), durante la transición a una modalidad de enseñanza diferente a la que había trabajado por 11 años, en un plan educativo de tipo presencial.

Empezaré por comentar que en el momento en que escuché que la Organización Mundial de la Salud (oms) había declarado una pandemia por la covid-19 me sentí muy angustiada por lo que se pudiera presentar. No obstante, sabía que debía continuar. Los sentimientos eran encontrados: tenía miedo por la enfermedad y el peligro de contagio, incertidumbre por todo lo que se escuchaba y ansiedad porque nos encontrábamos en la fase final del ciclo escolar, pues nuestra licenciatura tiene un plan anual. Hubo muchas dudas en la forma en que íbamos a continuar dando clases en asignaturas que tenían un enfoque cien por ciento clínico (atención de pacientes). Además del hecho de tener que cancelar las consultas con tratamientos dentales que estaban por terminar, entre otros. Pero también sabíamos en la Facultad que debíamos estar pendientes de las indicaciones.

En cuanto a las clases, aunque ya tenía cerca de 2 años trabajando con Google Classroom, debía buscar otra forma de comunicarme con los estudiantes. La verdad es que todos los grupos con los que me dirigí fueron solidarios y mostraron gran empatía por la situación en la que todos nos encontrábamos. Incluso, estuvieron muy pendientes entre ellos monitoreando sus dudas e inquietudes frente al cambio que llegaba. Durante la primera semana de confinamiento, dejé actividades a distancia, porque no tenía idea de cómo planear una clase en línea. Pero ya confinada totalmente en casa con mi familia, estaba consciente de que debía buscar la forma de dar clases en asignaturas teóricas, prácticas (laboratorio) y clínicas (atención de pacientes), pues aunque nos encontrábamos en la fase final del ciclo, había muchos puntos que debían ser repasados y considerados, sobre todo con los estudiantes de cuarto año que estaban a nada de terminar su formación universitaria y que, a diferencia de los otros años, debían terminarla a distancia. 
Fue así como busqué el apoyo y guía de dos profesores amigos. Con ellos establecí un grupo de trabajo colaborativo, en donde compartimos sentimientos de miedo, angustia e incertidumbre sobre la transición, pero también pudimos compartir información sobre las plataformas y softwares; incluso pasamos buena parte de los días planeando y probando algunas herramientas con el objetivo de conocerlas a profundidad para su uso en las clases.

Es preciso mencionar que todo esto tomó un rumbo diferente con el apoyo de la Universidad, que ofreció cursos, talleres, aulas virtuales y plataformas, entre otros, con los que dimos continuidad a los cursos. Para ese momento era importante pensar en la forma, las estrategias y los temas que debían ser presentados en las clases en línea; no sólo en las de tipo teórico, sino en las prácticas de laboratorio y clínicas.

Después de varias horas de investigación, inicié con Zoom, en donde pudimos continuar con asignaturas con enfoque clínico, a través del análisis de los casos previamente atendidos y, otras veces, con videos o presentaciones para reforzar los temas.

De manera similar trabajé con los grupos de asignaturas de laboratorio. Ahí sólo continuamos con la revisión de los temas y con la elaboración de algunas prácticas en casa, con modelos figurados o simulados. La ventaja fue que ya contaban con el material, sólo se hicieron ajustes en los trabajos de algunos estudiantes que no lo tenían al alcance, lo que era totalmente entendible pues habían dejado su material en donde rentaban o porque se habían confinado en otras casas. Mi estrategia fue compartir dicha situación con el grupo, para que aquellos que no harían la práctica pudieran enviar ejercicios, presentaciones, mapas, cuadros y/o ensayos, entre otros, que hicieran referencia al tema, para que comprendieran los contenidos. Afortunadamente, siempre existió ese espíritu universitario que permitió que se apoyaran entre todos.

Para ese momento, identifiqué que debía tener otro espacio para dar clases porque escuchaba que Zoom empezaba a fallar. Pregunté a un profesor que muy amablemente me ayudó con asesorías en Microsoft Teams. En un principio me costó mucho trabajo, pero en una tarde pude comprender y agradezco enormemente su apoyo. Tuve que avisar a los estudiantes que nos mudábamos a otra plataforma y aunque eso pudo generar molestia por el desconocimiento que implicaba su uso, aceptaron continuar, a pesar de que exigiera tiempo para conocerla y manejarla.

Es así como, con el paso de los días, fui dándome cuenta que la pandemia duraría un tiempo considerable. Por ello, me apoyé en lo que la Universidad nos brindó, los cursos, seminarios, talleres y demás, para formarme en el uso de las distintas plataformas y softwares. Allí compartí con otros profesores universitarios la incertidumbre por la situación, pero también la preocupación por formarnos en esta nueva modalidad de enseñanza, para que los estudiantes sintieran mucho apoyo en esta transición. 
Hoy por hoy, he visto una respuesta favorable de los estudiantes. Aunque siguen las dudas sobre la reincorporación y sobre la práctica clínica, hemos platicado e iniciado el ciclo escolar con muchas esperanzas de que en algún momento podremos regresar a las actividades presenciales, pero, por ahora, debemos continuar en esta modalidad.

Cabe señalar que también debí destinar un tiempo para enseñar el uso de las plataformas en las que se desarrollarán las clases, porque para ellos también es nuevo. Les he agregado tutoriales que yo misma he creado y les he dejado espacios para dudas en las mismas plataformas. Hemos comentado que ya nos encontramos mas adaptados, ellos a la forma de aprendizaje y yo a la de enseñanza. Continuamos con diversas actividades como revisión y análisis de casos clínicos, y con la elaboración de prácticas con materiales que tengan en casa. Así, trabajan de una manera diferente pero con el enfoque odontológico; además, como profesora, me ha llevado a ser más creativa.

Sé que aún vienen muchos retos, pero considero que nuestra Universidad siempre estará ahí para nosotros. Sin lugar a duda, estos momentos, con aciertos, errores y demás, nos han permitido generar experiencias, algunas tristes por las consecuencias de la pandemia, pero otras que nos han acercado más como comunidad y universitarios. Como todos sabemos, esta situación sin precedentes nos ha llevado a cambiar muchos hábitos y costumbres, no sólo personales, también académicos y sociales, pero pienso que es momento de continuar.

¡Gracias a cada uno de los estudiantes que aceptaron el reto! ¡Gracias, unAm, por todo el apoyo! ¡La unAm no se detiene!

\section{Cómo CITAR ESTE ARTículo}

* Castillo Sánchez, Hilda Fabiola. (2021, enero-febrero). La enseñanza universitaria en odontología durante la pandemia. Revista Digital Universitaria (RDU), 22(1). DOI: http://doi.org/10.22201/cuaieed.16076079e.2021.22.1.17 